\section{EMBRYRIDDLE \\ Aeronautical University}

SCHOLARLY COMMONS

\section{International Journal of Aviation,} Aeronautics, and Aerospace

\title{
Quantitative Examination and Comparison of Altimetry Rules-of- Thumb for General Aviation
}

Thomas A. Guinn

Embry-Riddle Aeronautical University - Daytona Beach, Thomas.Guinn@erau.edu

Follow this and additional works at: https://commons.erau.edu/ijaaa

Part of the Aviation and Space Education Commons, Aviation Safety and Security Commons, and the Meteorology Commons

\section{Scholarly Commons Citation}

Guinn, T. A. (2018). Quantitative Examination and Comparison of Altimetry Rules-of-Thumb for General Aviation. International Journal of Aviation, Aeronautics, and Aerospace, 5(3). https://doi.org/10.15394/ ijaaa.2018.1264

This Article is brought to you for free and open access by the Journals at Scholarly Commons. It has been accepted for inclusion in International Journal of Aviation, Aeronautics, and Aerospace by an authorized administrator of Scholarly Commons. For more information, please contact commons@erau.edu. 


\section{Quantitative Examination and Comparison of Altimetry Rules-of-Thumb for General Aviation}

\section{Cover Page Footnote}

The author would like to thank the two reviewers for thoughtful and insightful comments, which helped greatly improve the paper. 
When asked how to correct true altitude (TA) for non-standard temperatures, pilots (all levels) and even meteorologists will typically try to apply the well-known rule of thumb (ROT) for density altitude (DA). That is, the DA increases (decreases) by 120 feet for every degree warmer (colder) than standard at a given elevation/altitude (Lester, 2007; Pope, 2011). However, this ROT is not applicable to TA calculations and can lead to large errors if misused for this purpose. This misuse is certainly understandable because the DA ROT does provide a height correction for a given temperature difference, much like the ROT for TA. In fact, both ROTs are based on the same fundamental equation but with significantly different assumptions applied. The ROTs will therefore return largely different answers for most scenarios creating potentially unsafe situations.

While the ROT for DA is widely known, the ROT for TA presented here is much less known in the United States. In fact, it does not appear in any Federal Aviation Administration (FAA) documents related to weather. However, it is presented in Joint Aviation Authorities (JAA) weather training manuals (Oxford Aviation Services Limited, 2001) and can be found on several United Kingdom training websites and discussion forums (e.g., Cat3C.com). The ROT is based on guidance provided in the International Civil Aviation Organization (ICAO) operational procedures for altimetry correction (ICAO, 2006).

The purpose of this paper is to closely examine and compare the theory and assumptions behind the two ROTs to elucidate and quantify their differences. The end goal is to provide a resource to help flight instructors better explain their proper uses and more fully describe the limitations of each. Helping to improve general aviation (GA) pilots' understanding and ability to quantify the effects of temperature on altimeter, especially TA, may even help mitigate controlled flight into terrain (CFIT) accidents, which remain a significant problem for GA (Bailey, Peterson, Williams, \& Thompson, 2000; FAA, 2003; Landsberg, 2017; Shappell \& Wiegmann, 2003). We begin the paper with a background review of the underlying principles from which both ROTs are based. Next, we show the origins of the ROTs, describing in detail the assumptions of each. Lastly, even though DA and TA measure two different physical parameters, they are often confused so we examine the potential error introduced if one is mistaken for the other. 


\section{Background}

\section{Basis of Altimetry Equations: The Hydrostatic Equation}

Both the TA and DA ROTs originate from the hydrostatic equation, which simply describes the force balance between the vertical pressure gradient force and gravity (Guinn \& Mosher, 2015). When combined with the equation of state for an ideal gas, the hydrostatic equation can be expressed as:

$$
\frac{d p}{p}=-\frac{g}{R} \frac{d z}{T}
$$

where the independent variable is atmospheric pressure $(p)$, and the dependent variables are height $(z)$ and atmospheric temperature $(T)$. The constants, $g$ and $R$, represent the gravitational constant and the gas constant for dry air, respectively, which have values of $9.90665 \mathrm{~m} \mathrm{~s}^{-2}$ and $287.053 \mathrm{~J} \mathrm{~kg}^{-1} \mathrm{~K}^{-1}$ taken from the U.S. Standard Atmosphere (SA) (National Oceanic and Atmospheric Administration [NOAA], 1976).

Starting with the hydrostatic equation, our goal is to integrate (1) to derive a relationship for the height between two known pressure surfaces. For altimetry purposes, the two pressure surface values are typically an observed pressure (i.e., the pressure measured by the altimeter's internal aneroid barometer) and some reference pressure (i.e., the altimeter subscale). The crux of the problem then becomes specifying the atmospheric temperature structure between the two pressure levels, so we can complete the integration. The choice of assumptions in describing the vertical temperature profile is where the equations for DA and TA fundamentally differ. We describe each below.

Derivation of DA from the Hydrostatic Equation. Recall pressure altitude (PA) represents the height at which a given pressure occurs in the SA. Likewise, DA is the height at which a given density occurs in the standard atmosphere. The derivation of DA stems from the integration of (1) assuming a SA temperature lapse rate, where temperature changes linearly with height at a rate $L=-6.5^{\circ} \mathrm{C} \mathrm{km}^{-1}$ with a base temperature $\left(T_{o}\right)$ of $15^{\circ} \mathrm{C}(288.15 \mathrm{~K})$ (NOAA, 1976). By applying the SA temperature profile, we can easily integrate (1) from standard mean sea-level pressure, $p o=1013.25$ (29.921" $\mathrm{Hg})$, to the observed pressure, $p$, as shown in (2).

$$
\int_{p_{o}}^{p} d \ln p=-\frac{g}{R} \int_{0}^{h(p)} \frac{d z}{\left(T_{o}+L z\right)} .
$$


After completing the integration and solving for $h$, we obtain the equation for DA height $\left(h_{D A}\right)$. A more detailed derivation of the DA equation can be found in Guinn \& Barry (2016).

$$
h_{D A}(p, t)=\frac{T_{o}}{L}\left[\left(\frac{p}{p_{o}} \frac{T_{o}}{T}\right)^{-\frac{R L}{g+R L}}-1\right]
$$

The DA equation (3) provides the height at which a given temperature and pressure combination occur in the standard atmosphere. However, since temperature and pressure uniquely determine the density, (3) also provides the height at which a given density occurs in the SA, thus the name "density" altitude. Furthermore, when a value of $T$ is used that corresponds to the SA temperature for the input pressure, $p$, then (3) simply returns the PA. For this reason, the density altitude is most frequently defined as the PA adjusted for non-standard temperatures. Figure 1 shows DA as a function of pressure for three different temperature profiles. The center line uses a SA temperature profile, so the DA exactly equals PA for this case. The outside two lines use the same SA temperature profile but with a $\pm 20^{\circ} \mathrm{C}$ offset. By moving vertically along a constant pressure line, Fig. 1 describes how the DA changes with temperature from its SA value (i.e. the PA). We see that when the temperature is warmer (colder) than standard at a given pressure, the DA is slightly higher (lower), as expected.

It is important to note the DA equation provides no information regarding the actual atmosphere. It simply tells us where a given density (temperature and pressure combination) can be found in the SA. As pilots know, the real benefit of DA is that it provides a useful benchmark for flight performance calculations that rely on air density, such as thrust and lift. It does so by relating air density to an altitude in the standard atmosphere. Since density decreases with altitude in the SA, pilots know that higher values of DA indicate worsening aircraft performance, especially for takeoff. 


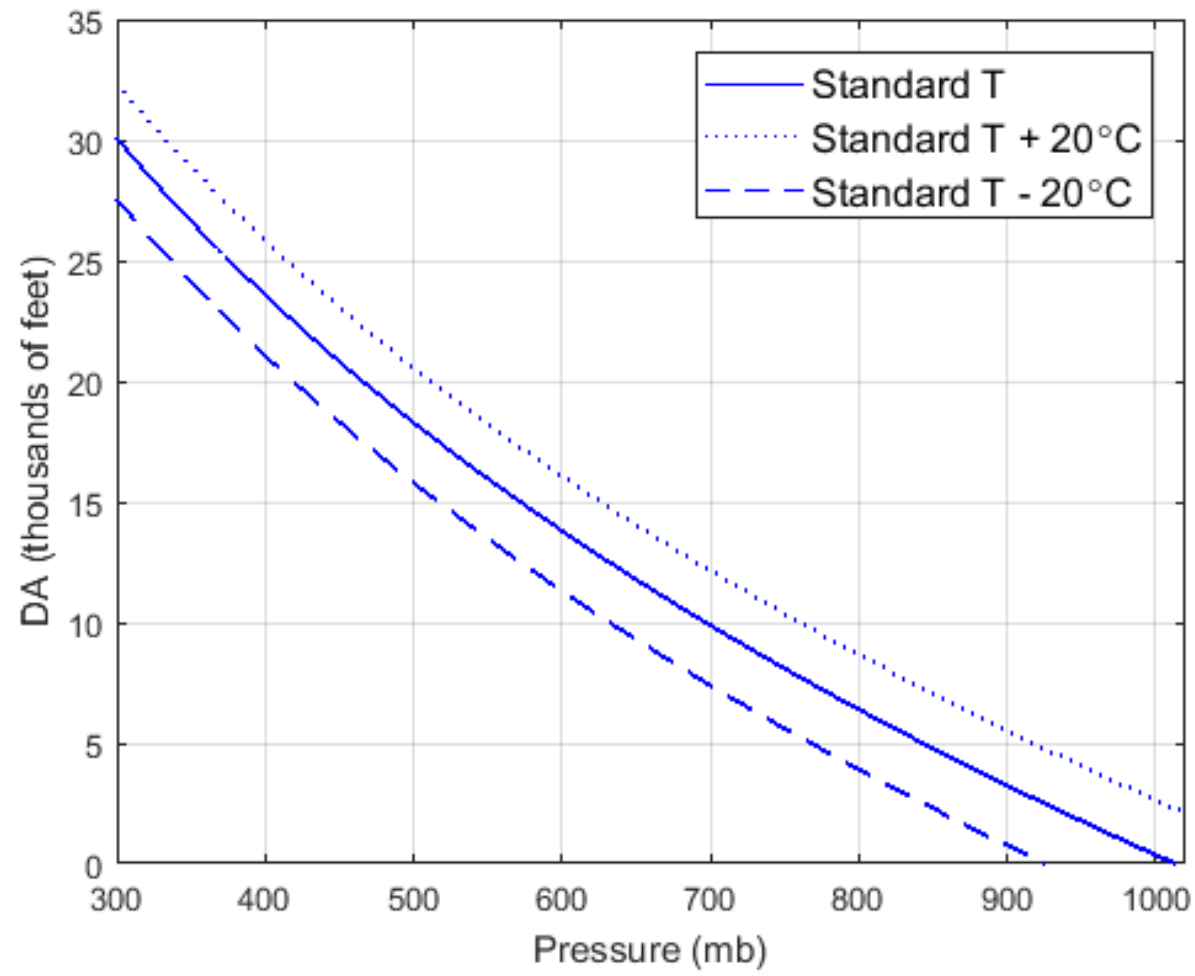

Figure 1. Change in density altitude (DA) with pressure assuming three different temperature profiles: 1) a standard atmospheric temperature (SA) profile, 2) an SA temperature profile $+20^{\circ} \mathrm{C}$, and 3) an SA profile $-20^{\circ} \mathrm{C}$. The SA profile is identically equivalent to the pressure altitude (PA).

Derivation of TA from the Hydrostatic Equation. In contrast to DA, TA depends on the mean temperature of the atmospheric layer between mean sea level and the aircraft. Since warm air is less dense than cold air, pressure decreases more slowly with height in a warm atmosphere as demonstrated in Fig. 2. Warmer than SA layers will cause the TA to be higher than indicated and vice versa. In contrast to DA, the calculation of TA must be based on the actual atmospheric temperature profile, for which no simple linear relationship with height exists. We must instead know (or approximate) the mean temperature of the atmospheric layer between the observed and reference pressure levels, $p$ and $p_{\text {ref }}$, respectively. This allows us to employ the mean value theorem to integrate (1) as shown in (4). Here we define

$\bar{T} \int_{p_{r e f}}^{p} d \ln p=-\frac{g}{R} \int_{0}^{h(p)} d z$. 


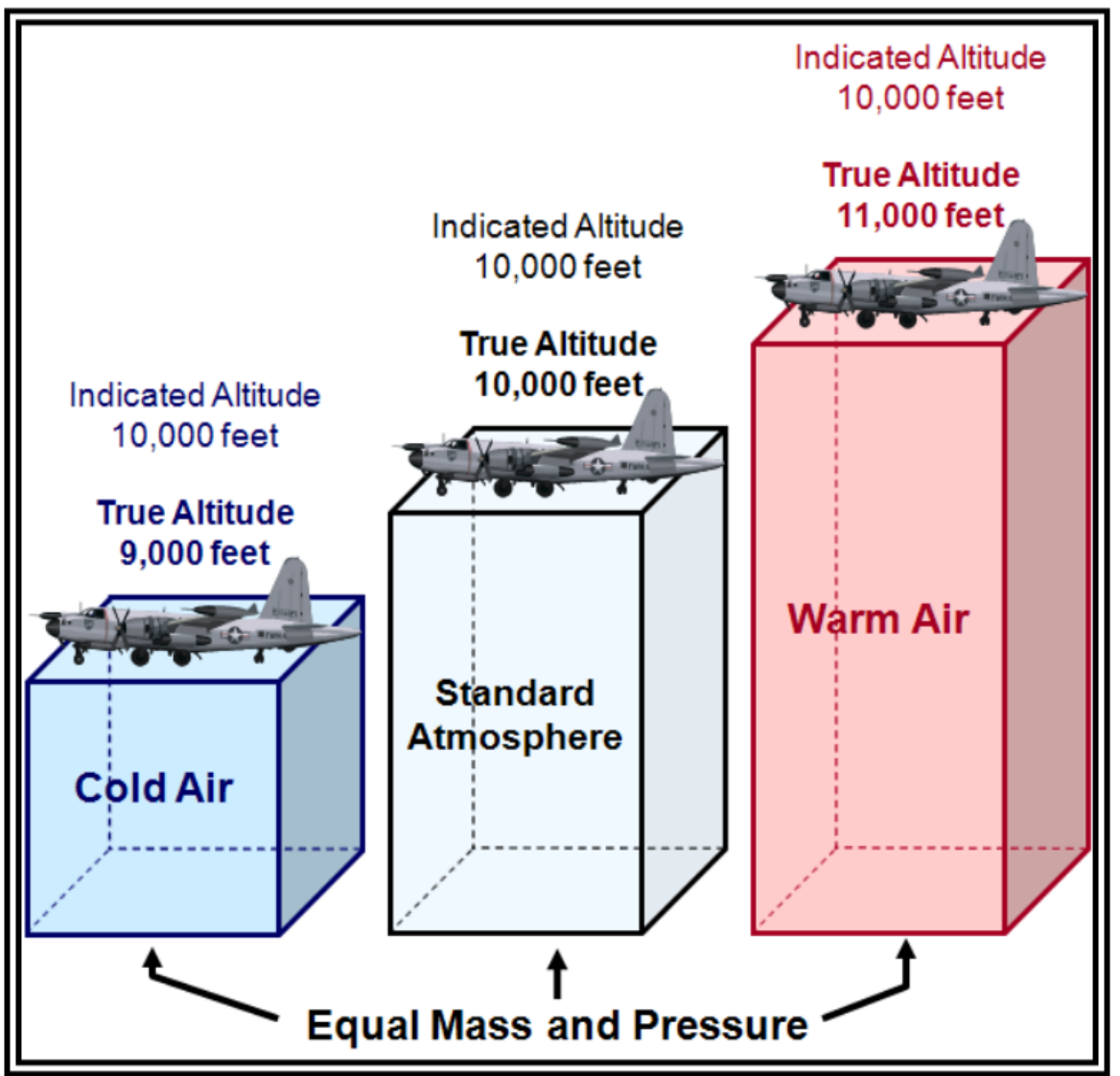

Figure 2. Depiction showing the impact of layer-mean temperature on altitude. Warm air is less dense than cold air, so it requires a higher column of air to exert the same surface pressure as the cold layer. However, altimeters are based on the standard atmosphere and will therefore report the same height for a given pressure level and reference pressure regardless of temperature. (Figure courtesy of FAA, 2016).

the height associated with the reference pressure (e.g., altimeter setting) to be zero. Upon completing the integration, we obtain an expression for the height of the layer between the two pressure surfaces. Notice the key difference between equations (2) and (4). In (4) there is no assumed linear temperature profile, i.e., $T=T_{o}+L z$.

$$
h(p)=\bar{T} \frac{R}{g} \ln \left(\frac{p_{r e f}}{p}\right) .
$$


Equation (5) is known as the hypsometric equation, and the variable, $h$, is frequently referred to as the "thickness" of the layer. The pressure, $p$, is the observed pressure, while the reference pressure can be any specified pressure depending on the height desired. If PA is desired (i.e., QNE), we use the standard datum plane value of $1013 \mathrm{mb}(29.92 " \mathrm{Hg}$ ). For true altitude (i.e., QNH), we use the current altimeter setting. Lastly, for absolute altitude (i.e., height above field elevation or QFE), we use the station pressure.

For meteorological applications, $h$ is typically calculated using a temperature profile obtained by a balloon-launched radiosonde or from numerical model data. However, rather than computing the mean temperature for an entire layer, equation (5) is applied by breaking the layer into several sub-layers with the mean temperature of each sub-layer determined using a simple mean. The thicknesses of the individual layers are then summed to obtain the total depth of the desired layer. This helps ensure greater accuracy in calculation because the simple mean is more accurate when applied to smaller layers. However, for aviation applications, temperature profiles aren't readily available in real time. Therefore, ROTs have been developed to approximate the TA by estimating the layer-mean temperature based on the outside air temperature at altitude. The development of the ROTs for both DA and TA are discussed next.

\section{Development of the ROTs}

ROT for DA. As mentioned earlier, the classic rule of thumb for DA is that the density altitude increases by 120 feet for every $1^{\circ} \mathrm{C}$ the temperature is warmer than standard for a given pressure altitude. To see where the ROT originates, we differentiate (3) with respect to temperature while holding pressure fixed, resulting in (6). By plotting (6) for a typical observed range of tropospheric temperatures, we see that although the expression appears complicated, the result is a linear relationship for the change in height with temperature.

$$
\left(\frac{\partial h_{D A}}{\partial T}\right)_{p}=\frac{T_{o}}{T}\left(\frac{R}{g+R L}\right)\left(\frac{p}{p_{o}} \cdot \frac{T_{o}}{T}\right)^{-\left(\frac{R L}{g+R L}\right)}
$$

The linear nature of (6) is easiest to see by evaluating pressure and temperature at their SA mean sea-level values, i.e., $p=p_{o}$ and $T=T_{o}$. In this case the slope reduces to a constant that closely resembles the desired ROT in value.

$$
\left(\frac{\partial h_{D A}}{\partial T}\right)_{p=p_{o}}=\left(\frac{R}{g+R L}\right)=118.6 \text { feet } /{ }^{\circ} \mathrm{C}
$$


In fact, whenever we use the SA temperature corresponding to the specified pressure in (6), the result will reduce identically to (7) as shown in the appendix. Equation (7) tells us how the DA will change with temperature from its standard value (i.e., the PA) for any specified tropospheric pressure. In addition, the equation tells us the ROT is consistent and accurate for all ranges of pressure values in the troposphere, where the SA lapse rate is $L$. Figure 3 shows the rate of change of DA with temperature for multiple pressure levels. As can be seen, the relationship is nearly linear at approximately 120 feet per $1{ }^{\circ} \mathrm{C}$. The same linear relationship can also be seen in Fig. 1 by noticing the distance between the three lines (when moving vertically along a constant pressure line) remains nearly unchanged regardless of pressure. That is, when moving at constant pressure, the distance between the outer and inner lines is approximately 4,800 feet corresponding to a temperature change of $40^{\circ} \mathrm{C}$, giving a rate of change of approximately 120 feet per $1^{\circ} \mathrm{C}$. We can now use this linear rate of change to estimate the total height correction necessary for PA to equal DA, which is the height correction (7) multiplied by the observed temperature difference. Since the rate of change is nearly constant, we can express the DA ROT as:

$$
\operatorname{CorD} A \approx 120\left(T-T_{S A}\right)
$$

The $\operatorname{CorDA}$ represents the amount of height correction (feet) you must apply to the PA to obtain the DA. Here $T$ is the observed temperature $\left({ }^{\circ} \mathrm{C}\right)$ and $T_{S A}$ is the SA temperature $\left({ }^{\circ} \mathrm{C}\right)$ for the PA. For example, if an aircraft were at an elevation of 5,000 feet, we would use the $T_{S A}$ for a PA of 5,000 feet, or approximately $5^{\circ} \mathrm{C}$. If the observed temperature at 5,000 feet, $T$, was $15^{\circ} \mathrm{C}$, the result would be a required correction of $+1,200$ feet.

ROT for TA. The ROT for TA is based on both (5) and ICAO procedures for altimeter corrections (ICAO, 2006). The ROT states the indicated altitude should be reduced by four percent for every $10^{\circ} \mathrm{C}$ the atmosphere is colder than the SA for a given PA. To understand this, consider the relative error (9) between the actual true altitude of the aircraft $(h)$ and the estimated true altitude of the aircraft as indicated by the altimeter $\left(h_{I A}\right)$. The indicated altitude is based solely on the altimeter equation and therefore assumes a SA temperature lapse rate.

$$
E \equiv\left(\frac{h-h_{I A}}{h}\right)
$$




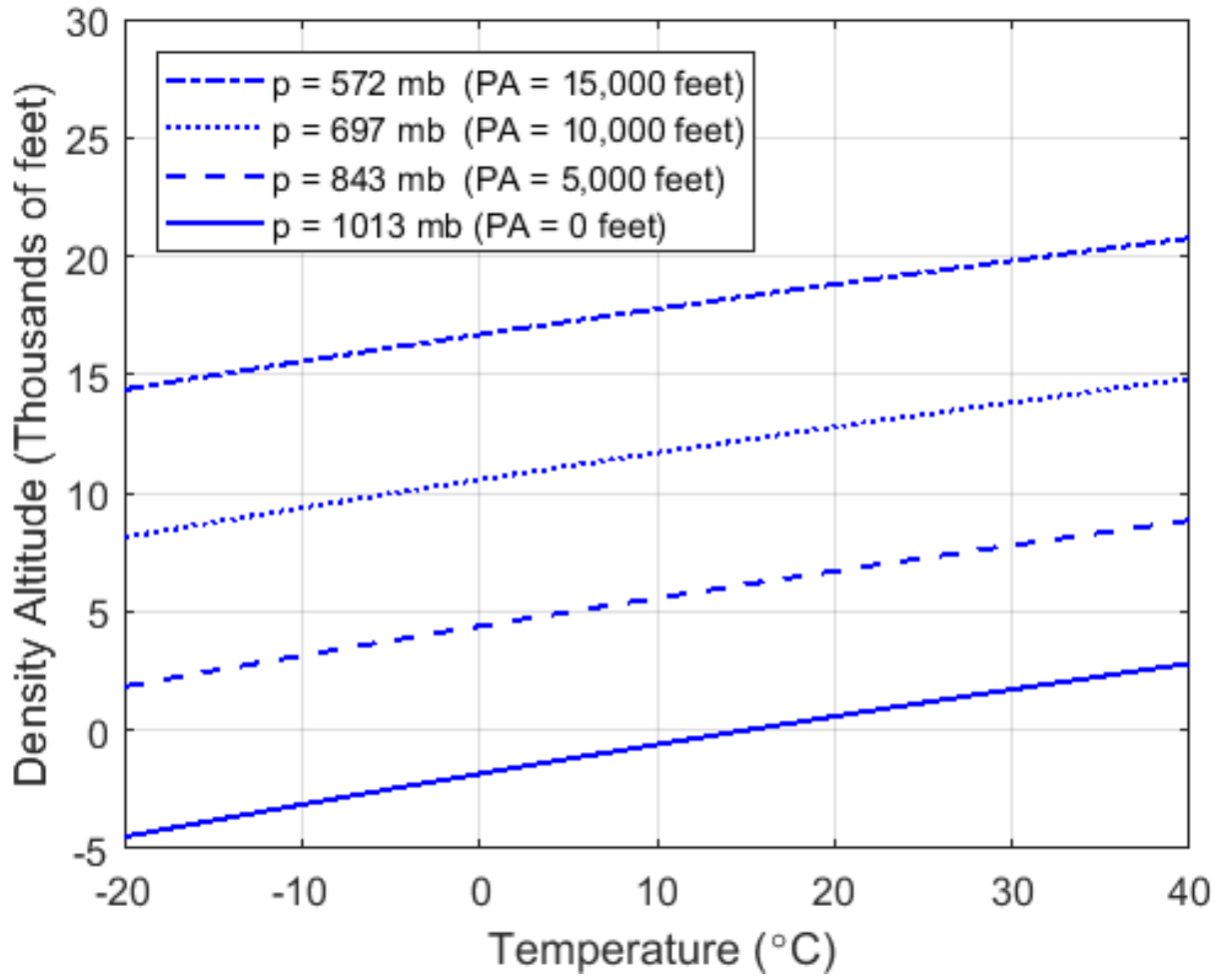

Figure 3. Change in density altitude with temperature for four pressure values corresponding to pressure altitudes of $0,5,000,10,000$ and 15,000 feet.

The error in (9) has the opposite sign of Guinn and Mosher (2015) because here we are interested in the necessary correction to indicated altitude such that we obtain true altitude; whereas, Guinn and Mosher (2015) calculated the additional height required for safe obstacle clearance, i.e., the correction to true altitude. Since the two heights in (9) are both based on the same reference pressure (i.e., the altimeter setting), we can apply the hypsometric equation (5) to relate the heights to the mean temperatures of the actual atmospheric layer and the mean temperature of the same layer in the standard atmospheric, $\bar{T}$ and $\bar{T}_{S A}$, respectively.

$$
E=\left(\frac{\bar{T}-\bar{T}_{S A}}{\bar{T}}\right)
$$

All temperatures in (10) must be measured in in Kelvins for the relationship in (9) to be valid. In addition, because Kelvins are used, the numerator will always be significantly smaller than the denominator, and 
therefore small changes in the denominator will have little effect on the entire quotient. This allows us to approximate the denominator with a single representative temperature of $250 \mathrm{~K}$ ( or $-23.15^{\circ} \mathrm{C}$ ), such that the relative error can be expressed as

$$
E \approx 0.004\left(\bar{T}-\bar{T}_{S A}\right) \text {. }
$$

From (11), we can now clearly see where the four percent ROT originates. When the observed mean temperature differs by $10^{\circ} \mathrm{C}$, the relative error will be exactly four percent. However, at this point, using the ROT is still a challenge because we rarely know the observed mean temperature. Use of the ROT requires yet another assumption to relate temperature to the layer-mean temperature. For this, we assume that not only does SA temperature vary linearly with height, but we also assume the observed atmospheric temperature varies linearly with height at the same SA lapse rate. If both are assumed linear, this means they can be directly related (with some small error due to height differences) to the outside air temperature and the SA temperature for the PA of the aircraft. That is,

$$
\left(\bar{T}-\bar{T}_{S A}\right) \approx\left(T-T_{S A}\right) .
$$

The assumption that the observed atmospheric temperature profile varies linearly with height is a significant weakness of the TA ROT, which is why the user should be cautious when using it. Consider the two temperature sounding profiles for Bismarck, ND shown in Fig. 4. Figure 4a shows a 12 UTC sounding in which a strong surface temperature inversion exists (solid red line). For comparison, the blue dashed line shows a linear temperature profile originating at the observed $700 \mathrm{mb}$ (approximately 10,000 feet) temperature. The linear temperature profile clearly misses the inversion and therefore produces a mean temperature that is too warm. As a result, the linear profile in Fig. 4a produces a height error of approximately $40 \mathrm{~m}$ (120 feet), and therefore the linear assumption produces a value that is too high compared to the observed true height of the layer. In contrast, the $00 \mathrm{UTC}$ sounding in Fig. $4 \mathrm{~b}$ has no inversion so the assumption of a linear temperature profile results in only a minor error of $6 \mathrm{~m} \mathrm{(18}$ feet) in this case. Because the observed atmosphere is rarely linear, the TA ROT is only a rough approximation. 
a)

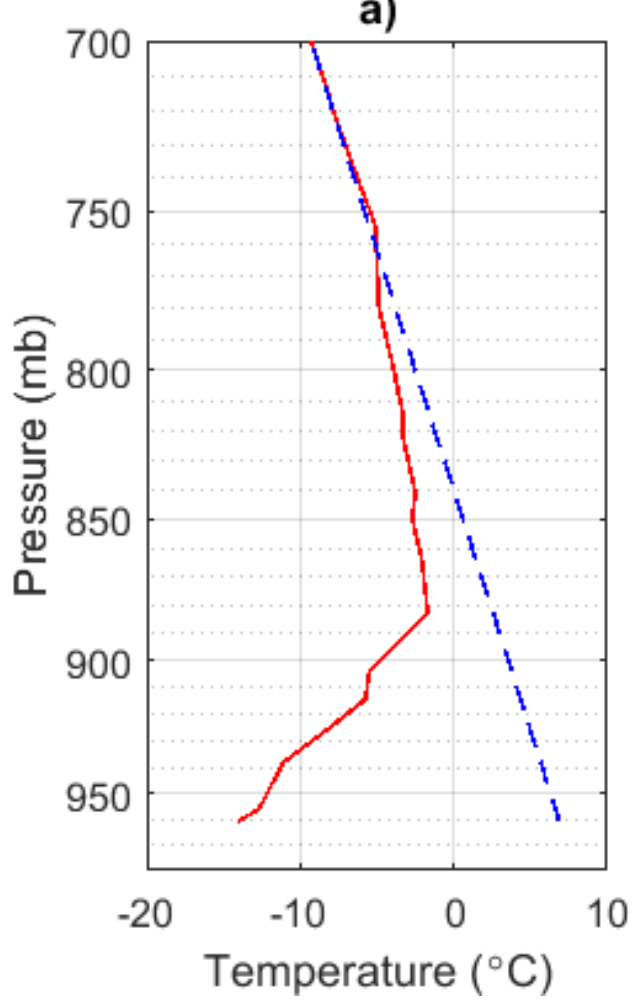

b)

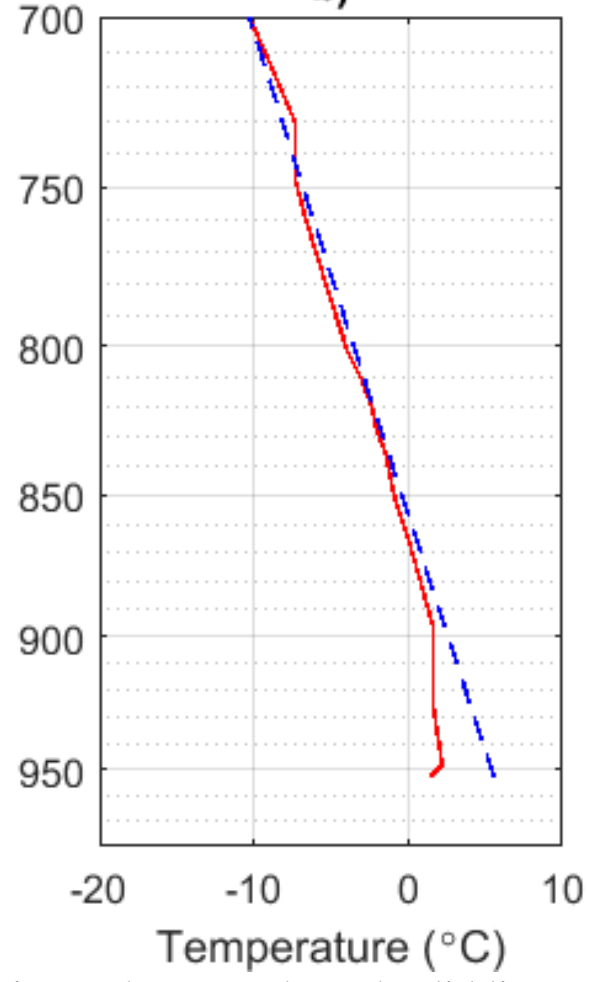

Figure 4. Temperature soundings from Bismarck, ND. The red solid line represents the observed temperature profile as determined from a radiosonde, while the blue dashed line is a linear temperature profile starting with the observed temperature at $700 \mathrm{mb}$. Fig. $4 \mathrm{a}$ is a morning sounding taken at 12UTC January 4, 2018, while Fig. 4b is an evening sounding taken at 00UTC January 8, 2018.

By substituting (12) into (11), we can use the relative error to estimate the amount of correction ( $\operatorname{CorTA}$ ) needed to be added to the indicated altitude to obtain the actual TA. That is,

$$
\operatorname{CorTA}=0.004\left(T-T_{S A}\right)\left(h_{I A}-h_{S f c}\right) .
$$

Note the error is only applied to the height above the terrain $\left(h_{s f c}\right)$. This is because the altimeter setting assumes a SA profile in the fictitious layer between the surface and mean sea level. Because of this, an altimeter with a properly set subscale will always read the runway elevation while on the airfield, so correcting for this height is unwarranted. This effect is shown in Fig. 5. Aircraft one is parked on the runway at 5,000 feet. Despite the temperature being 
$20^{\circ} \mathrm{C}$ below standard, the aircraft is experiencing no altimeter error while on the ground. Again, this is because the altimeter is calibrated to the SA and the fictitious layer beneath the surface is assumed to be SA as well. That is, the altimeter setting equation assumes a SA profile. In contrast, aircraft three, located above airport C (sea level), will experience an error of approximately $400 \mathrm{ft}$. This is because the entire atmospheric column of air above the runway is subject to the relative error. Lastly, for aircraft two, located over airport B (elevation 2,000 feet), only $60 \%$ of the atmospheric column is affected by the relative error, so the correction is smaller. Figure 5 also demonstrates that the TA ROT should only be applied at point locations. Calculating the necessary correction at one location and applying it to another could potentially result in significant error. For example, if aircraft one was to fly towards airport three, the aircraft would simply descend if attempting to maintain the same indicated altitude. However, if aircraft three continued towards airport one with no altimeter adjustment, it could result in a CFIT accident.

Using (13) we can now examine the case in Fig. 4a more closely. The temperature at $700 \mathrm{mb}$ (PA 9,878 feet) was $-9.3^{\circ} \mathrm{C}$ giving a deviation from SA of $-4.6^{\circ} \mathrm{C}$. The altimeter setting at the sounding site was approximately $30.01^{\prime \prime} \mathrm{Hg}$ such that the indicated altitude would have been 9,968 feet. Since the station elevation at Bismarck, ND is 1,686 feet, the total depth of atmosphere affected by the colder than SA temperature was 8,272 feet. Applying (12), the correction to TA for colder than SA temperature is approximately -150 feet. However, recall the assumption of a linear profile created a height error of nearly the same magnitude ( -120 feet). Thus, when accounting for both the height error introduced by assuming a linear temperature profile and the altimeter correction due to temperature being colder than SA, the total altimeter error for this case should have been approximately -270 feet. The ROT's assumption of a linear temperature profile therefore underrepresented the actual error by nearly half. This will happen whenever significant inversions exist, which frequently occur during early morning hours or near frontal boundaries. The important take-away is that the TA ROT should always be used cautiously because of the inherent assumptions. It is meant for increased situational awareness rather than operational decision making. 


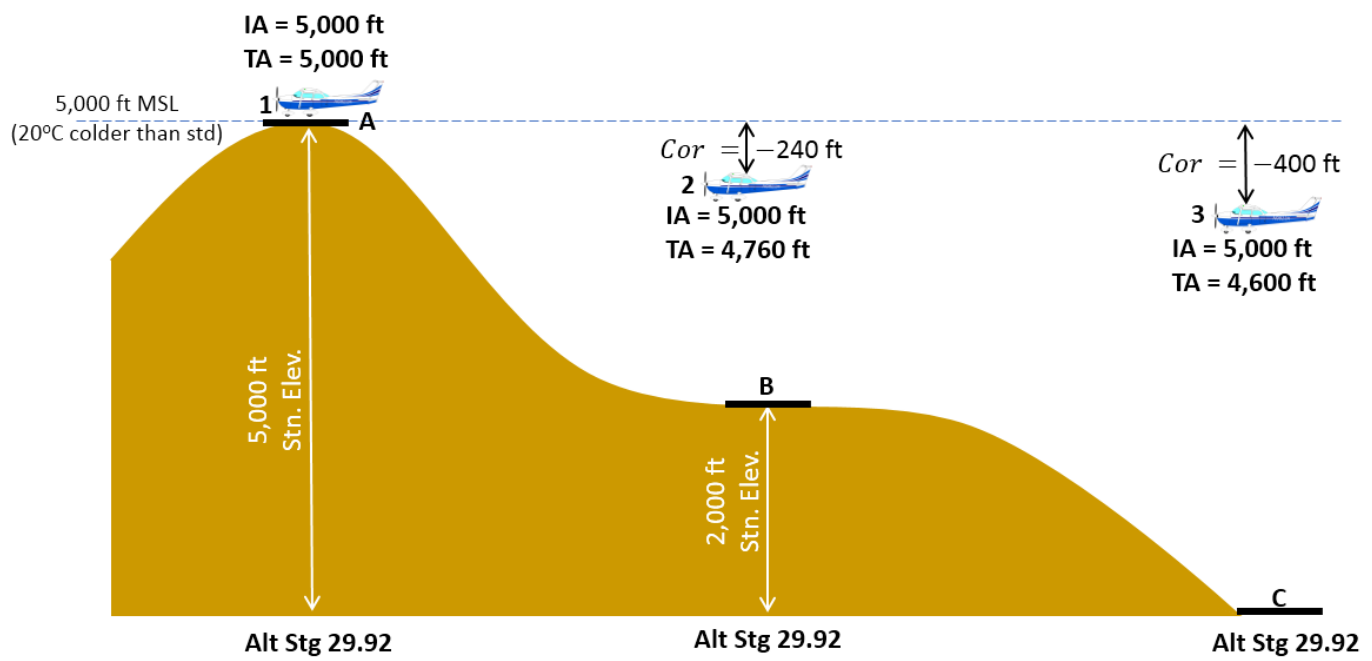

Figure 5. The altitude correction required to obtain true altitude from indicated altitude for aircraft departing from three different airports. Aircraft 1 is located directly at airport A (station elevation 5,000 ft), aircraft 2 is located directly above airport B (station elevation of 2,000 ft), while aircraft 3 is located directly above airport $\mathrm{C}$ (station elevation $0 \mathrm{ft}$ ). Assumptions: In all cases the outside air temperature at $5,000 \mathrm{ft}$ is $20^{\circ} \mathrm{C}$ colder than standard, the mean sealevel pressure is standard, and the altimeter subscales are correctly set. The difference in correction is caused by the depth of the layer over which relative error can exist.

\section{Methodology for Comparing ROTs}

To directly compare the two ROTs for illustrative purposes, we plotted the height correction $(\Delta h)$ versus the temperature deviation $(\Delta T)$ for both ROTs. In the case of TA, $\Delta h$ represents the height correction necessary for indicated altitude to provide TA. While for DA, $\Delta h$ represents the height correction necessary for the PA to provide the DA. Since the TA ROT depends on the depth of the layer over which the temperature deviation occurs, we chose representative depths of 5, 10, and $15 \mathrm{kft}$ because of their applicability to GA. Rather than mean sea-level heights, these values should be interpreted as the height difference between the aircraft's indicated altitude and the surface elevation directly below the aircraft.

In addition to defining what $\Delta h$ physically represents, we must also clearly define what the $\Delta T$ represents for the two ROTs. For the DA ROT, $\Delta T$ represents the temperature deviation at a given pressure level from the SA temperature for the same level. In contrast, for the TA ROT, $\Delta T$ represents the temperature deviation of the layer-mean temperature from the SA layer-mean temperature. 
This is the most fundamental difference between the two ROTs and the reason the two differ significantly. The ROTs were plotted over a temperature range of $15^{\circ} \mathrm{C}$ to $15^{\circ} \mathrm{C}$ with the results provided in Fig. 6 .

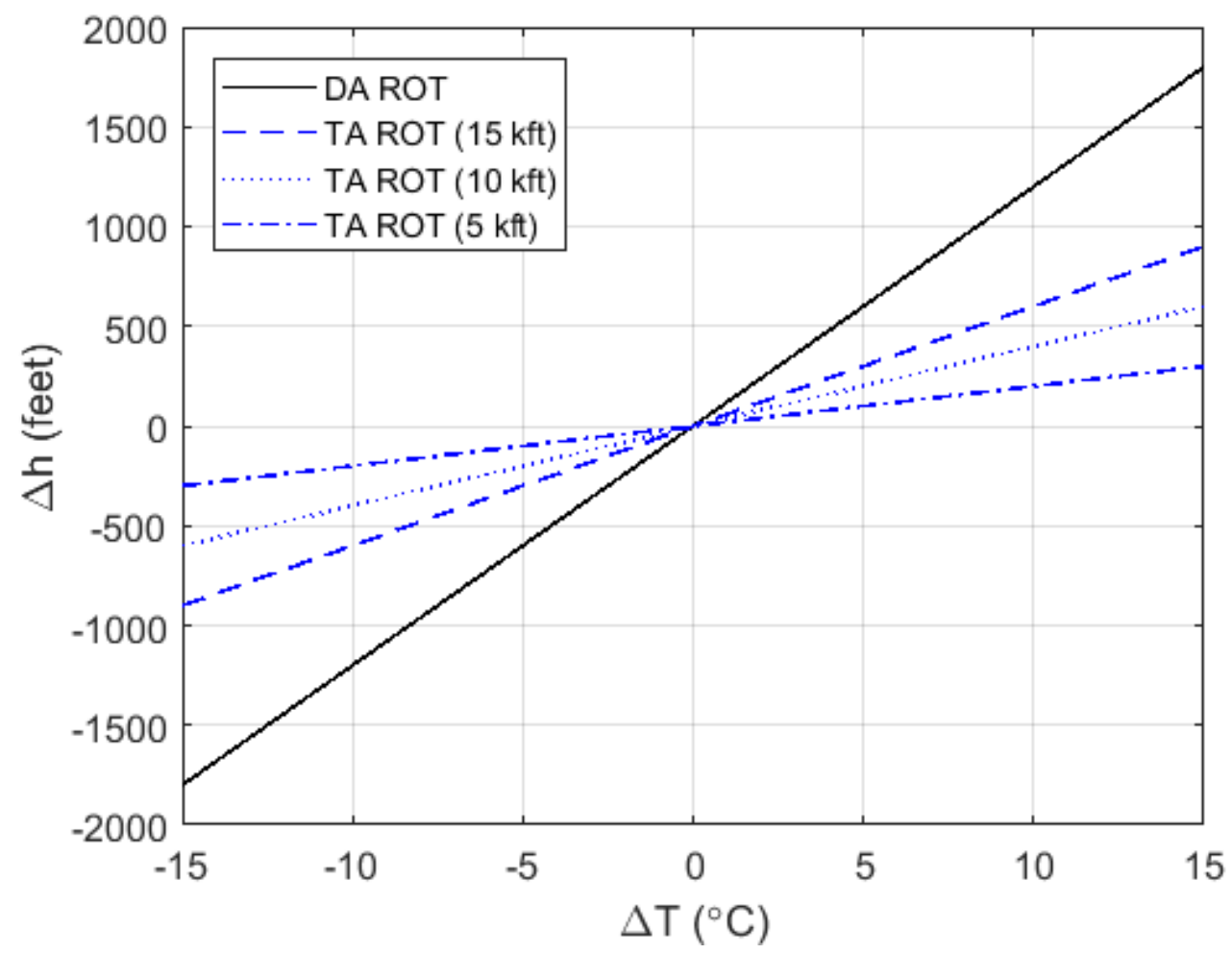

Figure 6. Simple comparison of the Rules of Thumb (ROT) for density altitude (DA) and true altitude (TA) showing the amount of height correction for a given deviation in temperature from standard atmosphere (SA). The TA ROT is applied to absolute altitudes of 15,000, 10,000 and 5,000 feet. (The DA ROT is independent of absolute altitude).

\section{Results of ROT Comparison}

By examining Fig. 6, we observe the DA ROT will overcorrect indicated altitude if misused for a TA correction. For example, when applied to an altitude of $5 \mathrm{kft}$, the DA ROT would overcorrect the TA ROT by a factor of nearly seven $(\sim 1,750$ feet vs $\sim 250$ feet $)$ for a temperature deviation of $-15^{\circ} \mathrm{C}$ colder than standard. Even for an indicated altitude of $15 \mathrm{kft}$, the DA ROT would still overcorrect by nearly a factor of two. While not a hazard, since overcorrection only adds an additional margin of safety, it clearly demonstrates the difference in the ROTs. A more dangerous situation would occur if using the TA ROT to estimate the necessary DA correction. 
Again, it is important to emphasize the two ROTs are not intended to be interchangeable. Despite the mathematical similarity of the two ROTs (i.e., they both represent a height correction for a given temperature difference), they are based on different assumptions regarding the atmospheric temperature profile and therefore serve different purposes. The comparison in Fig. 6 is strictly made to show the potential error if a pilot were to misuse one ROT for the other.

\section{Discussion and Limitations}

An important distinction between the ROTs is the range of $\Delta T$ values over which both are operationally useful. For the DA ROT, positive temperature deviations (temperatures warmer than standard) are most significant because they equate to poorer than normal aircraft performance. Thus, the right side of Fig. 6 has the most operational usefulness for DA. In contrast, for the TA ROT, negative temperature deviations are more critical because they indicate when the TA will be lower than indicated, which creates the dangerous potential for CFIT. Thus, the left side is of Fig. 6 has the most operational usefulness for TA

A limitation of the methodology is that we have restricted our discussion to altitudes applicable to GA flight activity. For higher altitude flights, the slope of the TA ROT approaches that of the DA ROT. In fact, at a flight level of 30,000 feet MSL, the two are ROTs produce identical results, although the physical interpretation of the resulting values would be significantly different. For the altitudes of GA flights, however, the two ROTs are never interchangeable and should never be used as such. For conciseness and ease of reference, Table 1 highlights and summarizes some of the key differences and limitations for the two ROTs as discussed in the paper.

The most important discussion point is that the TA ROT is just that, a ROT. It should only be used for enhanced situational awareness and improved understanding of risk. More importantly, it should never be used in lieu of official FAA/ICAO cold temperature error correction tables, such as those published in the Aeronautical Information Manual (FAA, 2017). As another alternative to a simple TA ROT, Guinn \& Mosher (2015) created maps of estimated altimeter error by computing the difference between model-derived heights and the height calculated using the altimeter equation. Because the data is model based, both current and forecasts of altimeter error are possible. Currently these maps only exist for North America, but they could be expanded to other parts of the globe. 


\section{Summary and Conclusions}

The ROTs for DA and TA are mathematically very similar in form in that they both provide a height correction for a given temperature deviation from standard. However, the fundamental assumptions of the derivations are significantly different and should be thoroughly understood before using in practice or taught in the classroom.

For the TA ROT, the required temperature deviation is intended to be representative of the layer-mean temperature difference between the observed atmosphere and the SA for the layer between the pressure measured by the altimeter's internal aneroid barometer and the pressure set in the altimeter subscale. Since the layer-mean temperature is rarely known in flight, we must approximate it by using the observed temperature at altitude and assuming a linear lapse rate. This assumption can introduce large errors, especially when surface or frontal inversions exist in the lower troposphere. In contrast, for DA the input temperature deviation represents the difference between the observed temperature and the SA temperature for the same pressure level. As a result, the ROTs produce significantly different results, especially for the altitudes at which most GA flights occur.

One final thought is that even if the TA ROT is not presented in GA training, flight instructors should nonetheless stress the qualitative relationship between non-standard temperatures and altimeter performance, especially as it relates to CFIT. Likewise, they should also emphasize the well-known DA ROT should never be used to estimate TA. Future research could include using model reanalysis data to create climatological maps of altimeter error. These could then be used for improved pilot training and increased awareness by showing students how the magnitude of altimeter error due to non-standard temperatures varies by location on a seasonal or monthly basis. 
Table 1

Comparison of True Altitude and Density Altitude Rules of Thumb (ROT)

Category

Fundamental

principle

Rule

Application

Temperature

information

required

Input

temperature

difference

Output height correction

Additional requirements

Weaknesses

\section{True Altitude ROT}

Hydrostatic balance

Cor $=0.004(\Delta T)\left(h_{I A}-h_{s f c}\right)$

Applied to the atmospheric

layer between two pressure

levels (observed pressure and

altimeter subscale)

Requires layer-mean temperature to be known or approximated.

Represents the difference between the observed layermean temperature and the standard-atmosphere layermean temperature of the same layer.

Represents the height to be added/subtracted to the indicated altitude to obtain true altitude.

Dependent on height difference between indicated altitude and terrain height.

Observed layer-mean temperature is approximated using a linear lapse rate, which can miss surface and frontal inversions.

\section{Density Altitude ROT}

Hydrostatic balance

$\operatorname{Cor}=120(\Delta T)$

Applied to a single pressure altitude.

Temperature assumed to the standard atmosphere linear temperature profile.

Represents the difference between the observed temperature at a given pressure level and the standard atmosphere temperature for the same pressure level.

Represents the height to be added/subtracted to the pressure altitude to obtain density altitude

None.

None. Accuracy is constant with a relative error of less than 1.2 percent. 


\section{References}

Bailey, L. L., Peterson, L. M., Williams, K. W., \& Thompson, R. C. (2000). Controlled flight into terrain: A study of pilot perspectives in Alaska. Final Rep. DOT/FAA/AM-00/28, US Dept. of Trans., 49 pp. Retrieved June 23, 2018 from

https://www.faa.gov/data_research/research/med_humanfacs/oamtechrepo rts/2000s/media/00_28.pdf.

Federal Aviation Administration. (2003). General aviation controlled flight into terrain awareness. Advisory Circular AC 61-134, Federal Aviation Administration, 18 pp. Retrieved June 23, 2018 from https://www.faa.gov/documentLibrary/media/Advisory Circular/ac61134.pdf.

Federal Aviation Administration. (2016, August 23). Aviation weather. Advisory Circular AC 00-06B. Federal Aviation Administration. Retrieved June 28, 2018 from https://www.faa.gov/documentLibrary/media/Advisory_Circular/AC_006B.pdf

Federal Aviation Administration. (2017, October 12). Aeronautical information manual. Federal Aviation Administration. Retrieved June 23, 2018 from https://www.faa.gov/air_traffic/publications/media/AIM_Basic_dtd_1012-17.pdf

Guinn, T. A., \& Mosher, F. R. (2015). Numerical model derived altimeter correction maps for non-standard atmospheric temperature and pressure. International Journal of Aviation, Aeronautics, and Aerospace, 2(2). https://doi.org/10.15394/ijaaa.2015.1060

Guinn, T. A., \& Barry, R. J. (2016). Quantifying the effects of humidity on density altitude calculations for professional aviation education. International Journal of Aviation, Aeronautics, and Aerospace, 3(3). https://doi.org/10.15394/ijaaa.2016.1124 
International Civil Aviation Organization. (2006). Document 8168 OPS/611 Aircraft Operations, vol. 1, Flight Procedures, 5th ed., Ch 4, Altimeter Corrections. Retrieved June 23, 2018 from http://code7700.com/pdfs/icao_doc_8168_vol_1.pdf

Landsberg, B. (2017, February 1). Safety pilot landmark accident: Vertical thinking. Aircraft Owners and Pilot Association (AOPA) website. Retrieved June 23, 2018 from https://www.aopa.org/news-and-media/allnews/2017/february/pilot/landmark-accident-vertical-thinking

Lester, P. (2007). Aviation weather (3rd ed.). Englewood, CO: Jeppesen. ISBN13: 978-0-88487-446-1.

National Oceanic and Atmospheric Administration. (1976). U.S. standard atmosphere, 1976. Document S/T 76-1562, 241p. Retrieved June 23, 2018 from https://ntrs.nasa.gov/archive/nasa/casi.ntrs.nasa.gov/19770009539.pdf

Oxford Aviation Services Limited. (2001). Joint aviation authorities air transport pilot's licence theoretical knowledge manual: 050 Meteorology (2nd ed.). Frankfurt, Germany: Jeppesen GmbH.

Pope, S. (2011, October). Density formula for calculating density altitude. Flying Magazine, 138(10). Retrieved June 21, 2018 from https://www.flyingmag.com/technique/tip-week/calculating-densityaltitude-pencil

Shappell, S. A., \& Wiegmann, D. A. (2003). A human error analysis of general aviation controlled flight into terrain accidents occurring between 1990 1998. Final Rep. DOT/FAA/AM-03/4, US Dept. of Trans., 25 pp. Retrieved June 23, 2018 from http://www.dtic.mil/dtic/tr/fulltext/u2/a417230.pdf. 


\section{Appendix}

\section{Change in Density Altitude with Temperature in a Standard Atmosphere}

Here we show the equation for the change in density altitude (DA) with temperature reduces to the same constant value when evaluated at standard atmosphere (SA) temperature values, $T_{S A}$, corresponding to specified pressure altitudes $\left(h_{P A}\right)$. First, we define the SA temperature as:

$$
T_{S A}=T_{o}+L h_{P A}
$$

However, $h_{P A}$ can be related to pressure through the altimetry equation (Guinn \& Mosher, 2015) given by (A2).

$$
h_{P A}=\frac{T_{o}}{L}\left[\left(\frac{p}{p_{o}}\right)^{-\frac{R L}{g}}-1\right] \text {. }
$$

By substituting (A2) into (A1), we obtain a simplified expression for the temperature in the SA as a function of pressure (rather than height) in the SA.

$$
T_{S A}=T_{o}\left(\frac{p}{p_{o}}\right)^{-\frac{R L}{g}}
$$

Finally, by substituting (A3) for $T$ into equation (6), both pressure and temperature cancel giving the desired constant value for the rate of change (at constant pressure) of DA with respect to temperature when evaluated at $S A$ temperatures.

$$
\left(\frac{\partial h_{D A}}{\partial T}\right)_{p}=\left(\frac{R}{g+R L}\right)
$$

